\title{
Eye Movement Targets Are Released from Visual Crowding
}

\author{
William J. Harrison, ${ }^{1}$ Jason B. Mattingley, ${ }^{1,2}$ and Roger W. Remington ${ }^{1}$ \\ ${ }^{1}$ School of Psychology and ${ }^{2}$ Queensland Brain Institute, The University of Queensland, St Lucia, Queensland 4072, Australia
}

Our ability to recognize objects in peripheral vision is impaired when other objects are nearby (Bouma, 1970). This phenomenon, known as crowding, is often linked to interactions in early visual processing that depend primarily on the retinal position of visual stimuli (Pelli, 2008; Pelli and Tillman, 2008). Here we tested a new account that suggests crowding is influenced by spatial information derived from an extraretinal signal involved in eye movement preparation. We had human observers execute eye movements to crowded targets and measured their ability to identify those targets just before the eyes began to move. Beginning $\sim 50 \mathrm{~ms}$ before a saccade toward a crowded object, we found that not only was there a dramatic reduction in the magnitude of crowding, but the spatial area within which crowding occurred was almost halved. These changes in crowding occurred despite no change in the retinal position of target or flanking stimuli. Contrary to the notion that crowding depends on retinal signals alone, our findings reveal an important role for eye movement signals. Eye movement preparation effectively enhances object discrimination in peripheral vision at the goal of the intended saccade. These presaccadic changes may enable enhanced recognition of visual objects in the periphery during active search of visually cluttered environments.

\section{Introduction}

Voluntary eye movements are crucial for efficient sampling of the visual environment. During fixation, objects at the fovea receive enhanced processing and are easily recognized, whereas those in the periphery are more difficult to identify, particularly when closely adjacent objects surround them, a phenomenon referred to as visual "crowding" (Bouma, 1970; Pelli and Tillman, 2008). This effect can be experienced by fixating first on the red cross and then on the blue cross in Figure $1 A$. Note that the letter "Y" is much harder to discern when fixating the red cross than the blue one, even though it is located an equal distance from fixation in the two situations.

Crowding has been assumed to reflect obligatory integration of visual features in early visual areas that represent a region of space which includes both target and nontarget stimuli (Parkes et al., 2001). The extent of this region-the "critical distance"scales with eccentricity, so that the zone of crowding becomes progressively larger as target stimuli are moved further into the periphery. Critical distance is approximated by Bouma's law as $0.5 \varphi$, where $\varphi$ is the eccentricity of the target (Bouma, 1970; Pelli and Tillman, 2008). The spatial extent of crowding can be used to approximate the minimum cortical distance between two objects necessary for accurate object recognition (Pelli, 2008).

Received Aug. 31, 2012; revised Dec. 11, 2012; accepted Dec. 20, 2012.

Author contributions:W.J.H., J.B.M., and R.W.R. designed research; W.J.H. performed research; W.J.H. analyzed data; W.J.H., J.B.M., and R.W.R. wrote the paper.

This research was supported by an Australian Research Council (ARC) Discovery Project awarded to R.W.R. and J.B.M. (DP0666772). J.B.M. was also supported by an ARC Australian Laureate Fellowship (FL110100103). Parts of these data were presented at the Australasian Experimental Psychology Conference in April, 2012. We thank T.S.A. Wallis for his assistance with analysis of data for Experiment 2.

The authors declare no competing financial interests.

Correspondence should be addressed to William J. Harrison at his present address: Schepens Eye Research Institute, Massachusetts Eye and Ear Infirmary, Department of Ophthalmology, Harvard Medical School, Boston, MA 02114. E-mail: willjharri@gmail.com.

DOI:10.1523/JNEUROSCI.4172-12.2013

Copyright $\odot 2013$ the authors $\quad 0270-6474 / 13 / 332927-07 \$ 15.00 / 0$
Attempts to reduce crowding using spatial cues to indicate the location of the crowded target have yielded mixed findings, and only modest improvements in target discriminability at best (Felisbert et al., 2005; Strasburger, 2005; Scolari et al., 2007; Yeshurun and Rashal, 2010). Thus, it has been suggested that crowding imposes a fundamental limit on conscious vision (Levi, 2008; Whitney and Levi, 2011).

It is well established that responses of visual neurons and perception of uncrowded targets can be modulated by extraretinal signals generated before an eye movement. For example, Moore et al. showed that activity related to an eye movement command in the frontal eye fields (FEF) alters the gain of V4 responses to stimuli presented at the goal of the intended eye movement (Moore and Armstrong, 2003; Moore et al., 2003; Moore and Fallah, 2004). Moreover, Tolias et al. (2001) showed that the receptive fields of V4 neurons shrink in size and shift toward the saccade goal just before a saccadic eye movement. Human psychophysical studies have shown that enhanced identification of uncrowded targets at the goal of a saccade (Remington, 1980 ) is substantially greater than that arising from visual cues alone (Deubel, 2008). Critically, these neural and perceptual effects are evident before the eyes begin to move, before any change in the retinal location of the target stimulus (Kowler et al., 1995; Deubel and Schneider, 1996; Moore and Fallah, 2004; Deubel, 2008).

The evidence reviewed here suggests that an extraretinal signal involved in saccade preparation (Wurtz, 2008) can enhance perception at the goal of an eye movement. Whether similar perceptual improvements before a saccade occur for crowded targets remains an open question. We therefore tested if visual crowding is reduced when a crowded stimulus is the target of an intended saccade.

\section{Materials and Methods}

Overview of experiments. In two experiments, we quantified changes in the magnitude and spatial extent of visual crowding during steady fixa- 


\section{MOVE YOUR EYES}
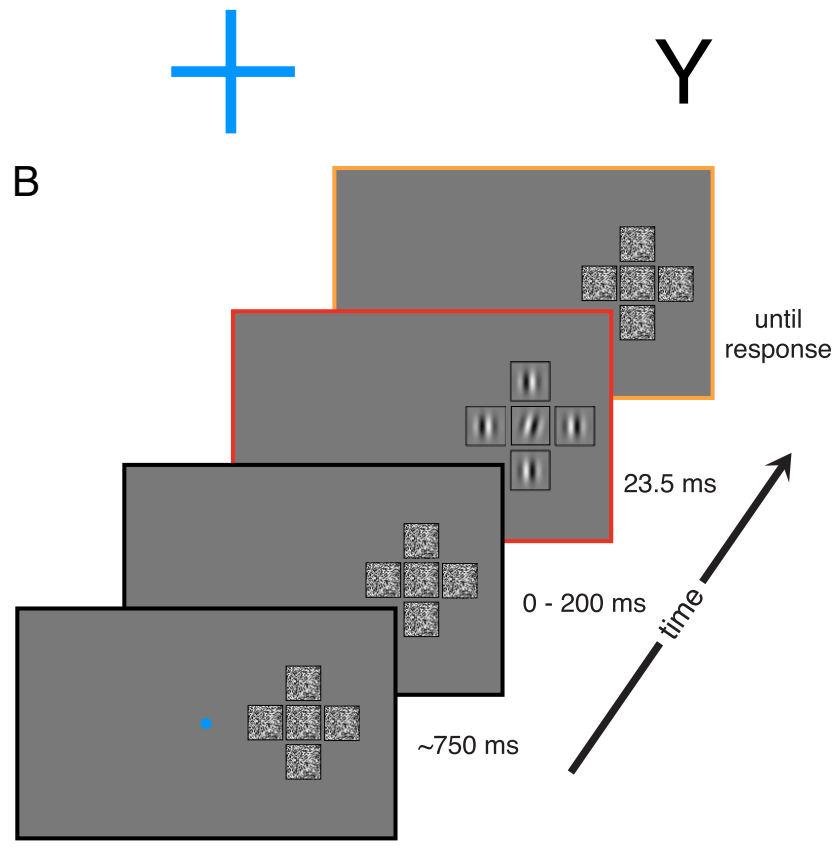

C

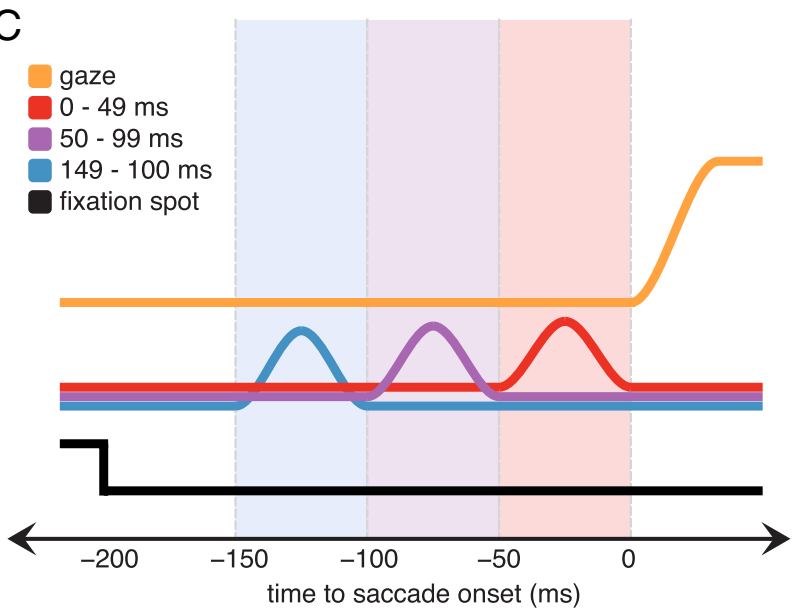

Figure 1. Demonstration of visual crowding and method used to test crowding before eye movements. A, Visual crowding for letter stimuli. In the upper row, the $Y$ in the word "EYES" is virtually impossible to identify while fixating the red cross. In the lower row, the $Y$ on its own is relatively easy to identify while fixating the blue cross, even though it is located at the same eccentricity as the $Y$ in "EYES" above. B, Sequence of displays used to quantify the magnitude of crowding before a saccade. At the offset of a blue fixation spot observers executed a saccade to the target and then reported the orientation of the central Gabor. If the fixation spot was red observers maintained fixation and performed the same task on the central Gabor. C, Schematic showing the timing of target displays relative to saccade onset. The saccade commences at time 0 , and negative times on the $x$-axis reflect the presaccade intervals over which target stimuli were presented. Saccadic latencies were recalculated continuously online. These latencies were used to determine target-saccade onset asynchronies, such that targets were presented with close to equal probability in each of three intervals before the saccade $(-149$ to $-100 \mathrm{~ms},-99$ to $-50 \mathrm{~ms}$, and -49 to $0 \mathrm{~ms}$; Hunt and Cavanagh, 2011). Dimensions of stimuli in $\boldsymbol{B}$ are not to scale.

tion and before a saccade toward a crowded target. Observers' basic task was to report the orientation of a peripheral target Gabor surrounded by four vertically oriented Gabors (Fig. 1B). Blocks in which central fixation was maintained ("no-saccade" trials) were intermingled with blocks in which observers executed a saccade to the target placeholder ("saccade" trials). Target presentation durations were brief $(<24 \mathrm{~ms})$ and, in saccade trials, were presented at varying times before the eye movement (Fig. 1C, Fig. 2B). Critically, because all orientation judgments were made before the eyes moved, the retinal locations of target and flanking stimuli were identical for the saccade and no-saccade conditions.

Observers. Five experienced psychophysical observers (one female) participated in each experiment. Two observers, including one author (W.J.H.), participated in both experiments. All observers had normal or corrected-to-normal vision and gave informed consent. The study was approved by The University of Queensland's School of Psychology Ethical Review Committee.

Materials. Participants sat with their head in a head and chin rest positioned $57 \mathrm{~cm}$ from a 20 inch Dell CRT monitor $(1600 \times 1200$ pixels, $85 \mathrm{~Hz}$ ) in Experiment 1 or $61 \mathrm{~cm}$ from a 17 inch Samsung CRT monitor $(1280 \times 1024$ pixels, $85 \mathrm{~Hz})$ in Experiment 2. Stimulus presentation, eye movement recording, and response collection were programmed using the Psychophysics Toolbox Version 3 (Brainard, 1997; Pelli, 1997) and Eyelink Toolbox extensions (Cornelissen et al., 2002) for MATLAB (MathWorks). Eye movements were recorded at $500 \mathrm{~Hz}$ with an EyeLink 1000 (SR Research) infrared eye tracker, calibrated using a 9 point calibration procedure.

Stimuli and procedure. Each trial began with a fixation spot (width $=$ $0.2^{\circ}$ ) in the center of a uniformly gray display. As shown in Figure $1 B$, the target and four flanker positions in Experiment 1 were indicated with black placeholders $\left(1^{\circ} \times 1^{\circ}\right)$. The target in Experiment 1 was $7.7^{\circ}$ to the right of the fixation spot, and the center-to-center distance between target and flankers was $1.3^{\circ}$. In Experiment 2, only the target position $\left(7^{\circ}\right.$ from central fixation) was indicated with a placeholder, and this placeholder was offset at target onset such that no borders were visible during target presentations. Target and flanking stimuli were Gabors (width = $1^{\circ}, 2 \mathrm{cpd}, 100 \%$ contrast) presented for $23.5 \mathrm{~ms}$. Immediately before target presentation, patches of white noise randomized with each screen refresh $(85 \mathrm{~Hz})$ were presented at positions corresponding to targets and flankers. In Experiment 1 only, the same dynamic white noise followed target and flanker presentation. Randomly from trial to trial, the combined target and flanker configuration was jittered vertically by $\pm 1^{\circ}$ to ensure that observers could not preprogram eye movements throughout each testing session.

A trial began after gaze was detected continuously for $500 \mathrm{~ms}$ within a $2^{\circ} \times 2^{\circ}$ region centered on the fixation spot. The fixation spot offset after a variable delay of 750-1250 ms (randomly drawn from a uniform distribution), cueing the observer either to make a saccade to the target (blue spot), or to remain fixated (red spot). Runs of saccade and nosaccade trials were alternated in blocks of 12, and testing always began with a saccade block to estimate target presentation times, as described below. In Experiment 1, observers completed 360 trials (180 saccade, 180 no-saccade) in a single testing session. In Experiment 2, each targetflanker separation was tested in a different session, and each observer completed a minimum of two sessions per target-flanker separation. The minimum number of trials completed by each observer in Experiment 2 was 3600 .

Using a method similar to that described by Hunt and Cavanagh (2011), the interval between the offset of the fixation spot and target onset was manipulated to maximize the number of trials presented in three time bins before saccade onset (Fig. 1C, Fig. 2B). We estimated the median saccadic latency of a saccade block after each saccade trial, and from this value subtracted 25,75 , or $125 \mathrm{~ms}$ to adjust the delay between fixation offset and target onset. During testing only, saccade latencies were taken as the time between fixation spot offset and the time at which the point of gaze shifted beyond $2^{\circ}$ to the right of screen center. Median saccade latencies were calculated separately for each saccade block. For the first trial of a block we used the median saccade latency from the previous block. For the first trial of the experiment, median saccade latency was manually set to $200 \mathrm{~ms}$. These time adjustments were pseudorandomized across a block of trials, such that there were four of each $(-25,-75,-125 \mathrm{~ms})$ per block of 12 . We then used these time adjustments in the next block of no-saccade trials to ensure stimulus timing was closely matched across saccade and no-saccade blocks. Saccade trial data 

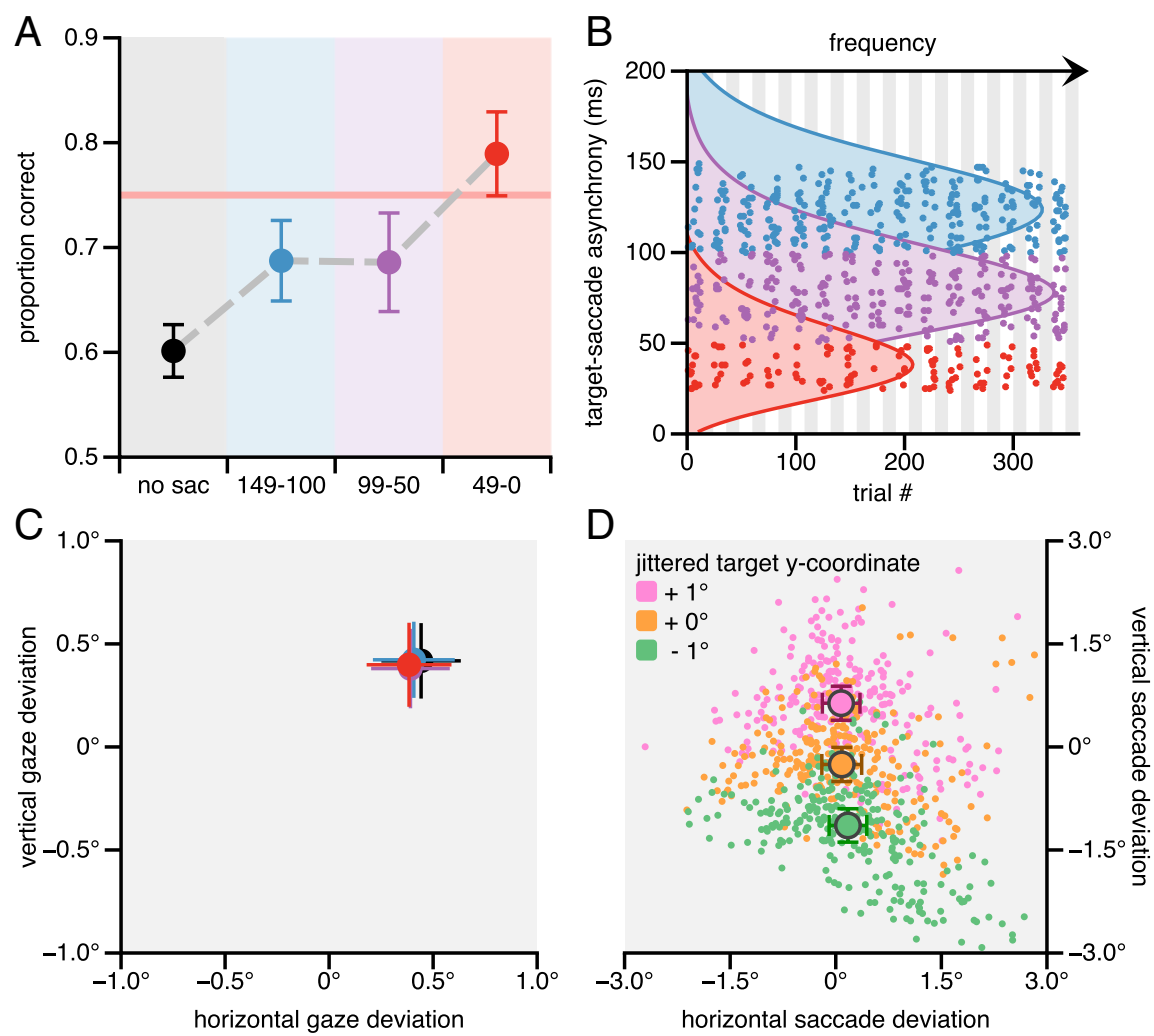

Figure 2. Influence of saccade preparation on visual crowding. $\boldsymbol{A}$, Mean percentage correct orientation judgments for a crowded Gabor target during central fixation (black symbol) and at $50 \mathrm{~ms}$ intervals before saccade execution (colored symbols). The horizontal red line indicates performance without flanking Gabors. $\boldsymbol{B}$, Frequency distributions of trials as a function of targetsaccade onset asynchrony. Target onset was timed to yield an approximately equal number of observations across three epochs (colored frequency distributions), and trials were screened and divided int $050 \mathrm{~ms}$ time bins (individual points). Only trials in which the target-saccade latency was $>24$ ms were included (i.e., included trials were exclusively those in which the target disappeared before the eyes moved). C, Graph showing mean gaze deviation from screen center during target presentation. Overlapping symbols show that observers maintained fixation close to the screen center in both no-saccade (black symbol) and saccade (colored symbols) trials. $\boldsymbol{D}$, Mean saccade endpoints corresponded to each of the three jittered target locations (see Materials and Methods). Observers executed eye movements toward the crowded targets with high accuracy, but saccadic errors were generally radially dispersed. Error bars indicate 1SEM. $n=5$.

were sorted into bins off-line according to the recorded target-saccade onset asynchrony, using a velocity of $30 \%$ s and acceleration of $8000 \% \mathrm{~s}^{2}$ as criteria for saccade onset. Following target presentation, there was a delay of $500 \mathrm{~ms}$ before observers were able to indicate the orientation of the target Gabor (rotated left or right off vertical; unspeeded, two-alternative forced-choice judgment).

Before testing, each observer completed a threshold procedure in which we established the minimum orientation to yield $75 \%$ correct identification of an unflanked target Gabor. We took the average orientation yielded by two interleaved QUEST procedures (40 trials each; Watson and Pelli, 1983). The target was presented after a delay of between 12 and $200 \mathrm{~ms}$ (randomly drawn from a uniform distribution) following the offset of the fixation spot. Observers were required to maintain steady fixation throughout threshold trials and gaze was monitored on-line. All other trial details during this threshold procedure were as described above.

Statistical analyses. To quantify changes in performance over time and across different target-flanker separations (Experiment 2), we used a permutation analysis described by Rolfs et al. (2005, 2011). Expected performance under the null hypothesis (i.e., that data within each targetflanker condition are temporally invariant) can be estimated by randomly permuting the observed data across time. These permutations were achieved by randomly re-assigning each response to a time bin (without replacement), creating a surrogate time course of data for each individual, from which we derived an average surrogate time course. This procedure was repeated 1000 times to yield a distribution of surrogate means for each time bin, representing an estimate of null performance across time. Using the distributions of permuted data, we calculated 95\% confidence intervals around estimated null performance, such that actual data falling beyond these intervals represent significant changes in performance across time. To verify this analysis, we bootstrapped data from each condition to estimate the distribution of the means (Efron and Tibshirani, 1993). In this case, for the number of observations in a given condition for each observer, we randomly sampled data from that condition (with replacement) to create bootstrapped means. By repeating this procedure 1000 times we created a distribution of means from bootstrapped data. From these distributions, we derived confidence intervals around the observed means, where two means falling outside each other's confidence intervals represents a significant change in performance. This analysis yielded the same significant changes over time as the permutation method.

Curve fitting and critical distance calculations followed previously described analyses (Scolari et al., 2007; Yeshurun and Rashal, 2010). Proportion correct data were modeled using a function with the following equation: $p c=a\left(1-e^{(-s(d-i))}\right), d>i$, where $p c$ is proportion correct, $a$ is the asymptote, $s$ is the scaling factor, $d$ is the target-flanker separation, and $i$ is the $x$-intercept. We repeated this procedure for each set of bootstrapped data (see above) to derive the confidence intervals. Critical distance, $c$, was calculated by: $c=i-\ln$ $(0.1) / \mathrm{s}$.

We quantified oculomotor precision by fitting an ellipse to saccade endpoints using custom code in MATLAB. We first found the $x-y$-coordinate of the center of all saccade endpoints, then, unconstrained, found the points in 2D space that created the center-to-edge distances of an ellipse comprising 95\% of all points. The axes of the ellipse were calculated by finding the two most extreme pairs of $x-y$ coordinates on the circumference of the ellipse in horizontal and vertical space.

Eye trace filtering. Trials were excluded if (1) fixation drifted $>2^{\circ}$ in any direction from the fixation spot; (2) a saccade $>2^{\circ}$ in amplitude was executed on a no-saccade trial; (3) probe onset occurred earlier than 150 $\mathrm{ms}$ or later than $24 \mathrm{~ms}$ before a saccade (with the exception of the analysis presented in Fig. 5, see text); (4) the target was presented during an eye-blink; and (5) a saccade endpoint was $>2^{\circ}$ from the target in Experiment 1 , or $4^{\circ}$ in Experiment 2. In total, 1373 trials $(76 \%)$ were included in Experiment 1, and 15,353 trials (79\%) were included in Experiment 2.

\section{Results}

\section{Experiment 1}

We first compared discrimination accuracy for a crowded target to which observers made a saccade with discrimination accuracy for the same target when observers instead maintained fixation centrally. The orientation of the target Gabor was adjusted for each observer using a staircase procedure (Watson and Pelli, 1983) to yield $75 \%$ correct responses for targets presented in isolation (i.e., unflanked) when no saccade was planned (see Materials and Methods). As expected, when no saccade was planned the flanking stimuli impaired orientation judgments for the target Gabor, such that observers' discrimination accuracy fell significantly to 
$60 \pm 2 \%$ (mean \pm SEM; two-tailed single sample $t$ test against 75\%: $t_{(4)}=5.93, p=$ 0.004; Fig. 2A).

When observers prepared a saccade to the crowded target, orientation judgments improved markedly in the interval between the signal to saccade to the crowded target and the initiation of the saccade (Fig. 2A). When the target and flankers appeared during the $50 \mathrm{~ms}$ immediately before saccade onset, orientation judgments were just as accurate as when the target was presented alone (unflanked) with no planned saccade. During this same $50 \mathrm{~ms}$ epoch before saccade onset, orientation judgments were also significantly more accurate than in nosaccade trials (two-tailed paired samples $t$ test, $\left.t_{(4)}=4.29, p=0.013\right)$. We screened data from the saccade condition to include only those trials in which the target was offset before the eyes moved (Fig. 2B). Thus, throughout the presaccadic interval, target and flanker stimuli always appeared at exactly the same retinal locations as in the no-saccade trials (Fig. $2 C$ ). As shown in Figure $2 D$, saccades were accurate despite the presence of flanking elements, and saccade endpoints were radially dispersed. The proportion of trials excluded due to saccade error was 5.2, 5.0, and 5.3\% for the $0-49,50-99$, and 100-149 $\mathrm{ms}$ presaccade conditions, respectively. These values were statistically indistinguishable (pairwise comparisons, all ps $>0.69$, uncorrected), ruling out the possibility that improved performance in the final time bin was artificially inflated by our saccade accuracy exclusion criterion.

To summarize the results of Experiment 1, immediately before a saccade, orientation discrimination accuracy is significantly improved for a crowded stimulus that is the target of an impending saccade. This release from crowding before a saccade yielded target judgments that were just as accurate as those made for unflanked targets at the same peripheral location in the nosaccade condition.

\section{Experiment 2}

Having established that visual crowding is significantly reduced when a peripheral stimulus is the target of a saccade, we next tested whether the critical distance of crowding around a target is also reduced just before an eye movement. A reduction in critical distance would suggest eye movement signals interact with the compulsory averaging of visual features (Pelli, 2008; Pelli and Tillman, 2008), effectively enhancing object discrimination in peripheral vision at the goal of the intended saccade. There are conflicting reports as to whether advance information reduces the critical distance of crowding in the absence of eye movements, despite improvements in target identification accuracy (Felisbert et al., 2005; Strasburger, 2005; Scolari et al., 2007; Yeshurun and Rashal, 2010). In a second experiment, therefore, we tested orientation discrimination for a peripheral target located at $7^{\circ} \mathrm{ec}-$ centricity, and surrounded by nontarget stimuli across a range of target-flanker separations. As in Experiment 1, observers either made a saccade to the target, or remained fixated at the center of the display.

Figure 3 shows the accuracy of target orientation judgments as a function of the time to saccade onset, plotted separately for each target-flanker separation. As expected, orientation judgments became progressively more accurate with increases in target-flanker separation (Bouma, 1970). When target and flankers were separated by $1^{\circ}, 1.5^{\circ}$, or $2^{\circ}$, accuracy peaked in the final $50 \mathrm{~ms}$ before the saccade (purple, blue, and green plots, respectively). In contrast, accuracy remained unchanged at target-flanker separations of 3.5 and $5^{\circ}$ (orange and red plots, respectively).

To quantify these changes in target identification accuracy across time, we used a permutation analysis to simulate expected performance under the null hypothesis of no change in accuracy across time (Rolfs et al., 2011; see Materials and Methods). In each plot in Figure 3, the dark lines show chance performance across time at each target-flanker separation, and the shaded regions the $95 \%$ confidence intervals around these simulated means. Points falling beyond the shaded regions indicate significant changes in accuracy. Improved performance just before a saccade was significant when target and flankers were separated by $2^{\circ}$, and fell just short of being significant when target and flankers were separated by $1^{\circ}$ or $1.5^{\circ}$. We verified these analyses using conventional bootstrapping methods to estimate confidence intervals (Efron and Tibshirani, 1993; see Materials and Methods), which yielded the same significant changes over time as described for the permutation tests. Together, the results reveal that presaccadic perceptual benefits for the $7^{\circ}$ target were not equal across all flanker separations, but were limited to the smallest separations, with marked improvement when flankers were separated from the target by $2^{\circ}$.

To estimate the critical distance of crowding we computed exponential fits to the accuracy data across target-flanker separations, separately for no-saccade trials and for saccade trials in which the target was presented within $50 \mathrm{~ms}$ before saccade execution (see Materials and Methods). Figure 4 shows the resulting functions plotted separately for no-saccade trials (gray) and saccade trials (maroon). By convention (Scolari et al., 2007; Yeshurun and Rashal, 2010) the critical distance of crowding is defined as $90 \%$ of the asymptotic value for each function (see Materials and Methods). The critical distance for no-saccade trials was estimated at $3.7^{\circ}$. Expressed as a ratio of target eccentricity, this yields a critical distance of $0.53 \varphi$, conforming well to Bouma's law (Bouma, 1970; Pelli and Tillman, 2008). In contrast, critical distance during the final $50 \mathrm{~ms}$ before a saccade was estimated at $2.0^{\circ}$, or $0.28 \varphi$, a $47 \%$ decrease in the critical distance observed during fixation.

In absolute terms, the critical distance for saccade trials indicates that flankers interfered with identification when they appeared within $2^{\circ}$ of the target stimulus. This critical distance estimate is consistent with the accuracy data presented in Figure 

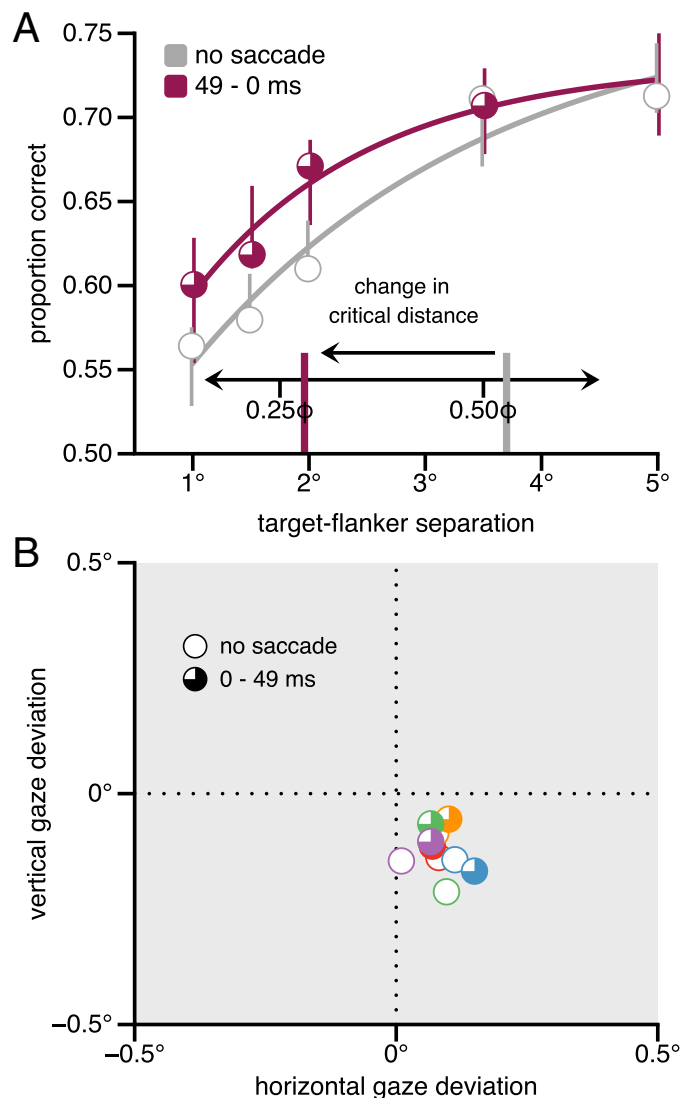

Figure 4. Change in the critical distance of crowding just before a saccade. $A$, Critical distance of crowding when no saccade is planned (gray fitted curve and vertical line) and in the final $50 \mathrm{~ms}$ before a saccade (maroon fitted curve and vertical line). The upper $x$-axis shows target-flanker separation as a proportion of the target eccentricity, $\varphi$. Error bars indicate $95 \%$ confidence intervals of the curves, derived from standard bootstrapping procedures (see Materials and Methods; Efron and Tibshirani, 1993). B, Horizontal and vertical gaze position during target presentation, shown as separate colored disks for the no-saccade condition (open symbols) and the $0-49 \mathrm{~ms}$ presaccade condition. Colors denote target-flanker separations as in Figure 3. Error bars have been omitted for clarity.

3: while accuracy improved significantly when flankers were $2^{\circ}$ from the target, no statistically reliable improvements in orientation judgments were observed when target-flanker separations were $<2^{\circ}$. The influence of saccade preparation on the critical distance of crowding cannot be attributed to differences in the retinal positions of target and flanker stimuli in saccade versus no-saccade trials. Horizontal and vertical gaze position during target presentation were matched across no-saccade trials and trials in which the target was presented in the final $50 \mathrm{~ms}$ before the saccade (Fig. 4B).

In line with studies showing eye movement centers influence visual processing of uncrowded stimuli (Corbetta et al., 1998; Moore and Armstrong, 2003), we propose that oculomotor programming interacts with early visual processes to alleviate crowding. If the reduction in the critical distance of crowding is due to spatially precise selection of the target location immediately before a saccade, then there should be a tight relationship between critical distance and the precision of oculomotor selection. To test this hypothesis, we plotted the accuracy of saccade landing points relative to the target position for all observers (Fig. 5A). The red and purple points show mean saccadic deviation from the target center when flankers were separated from the target by $1^{\circ}$ or $5^{\circ}$, respectively. These points fell the same distance from target center, revealing that oculomotor accuracy was unaffected by target-flanker separation. Each black spot in Figure $5 A$ repre-

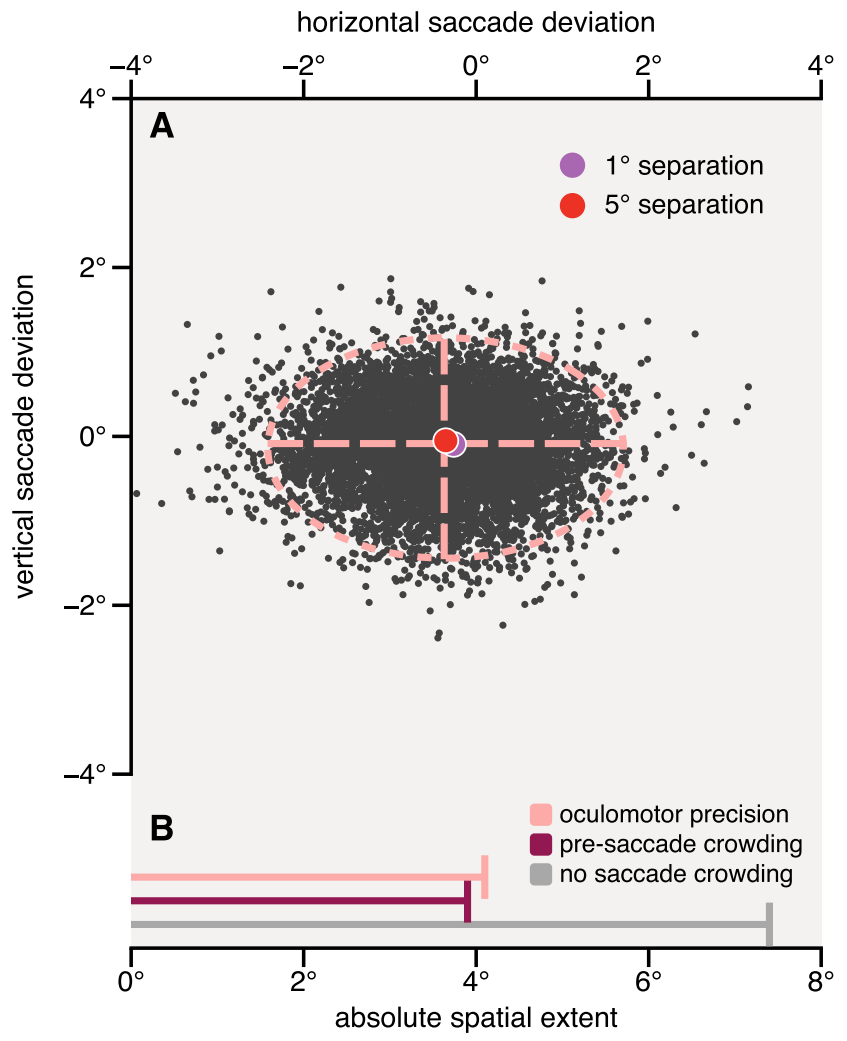

Figure 5. Relationship between oculomotor precision and the critical distance of crowding $A$, The horizontal and vertical deviations of 8153 saccade endpoints obtained from five observers are plotted on the $x$ - and $y$-axes, respectively. Position $0^{\circ}, 0^{\circ}$ represents the target center, and the target Gabor extended from $-0.5^{\circ}$ to $+0.5^{\circ}$ on both axes. Saccades with negative $x$-values fell short of the saccade target center, and saccades with positive $x$-values overshot the target center. An ellipse was adjusted to fit $95 \%$ of the saccade endpoints. As shown by the ellipse and its axes, saccadic precision was radially biased. The purple and red points show average deviations of saccade endpoints to targets with flankers at distances of 1 and $5^{\circ}$, respectively. There was no difference between these points; the proximity of flankers to the target did not interfere with oculomotor selection. $\boldsymbol{B}$, The spatial extent of observers' oculomotor radial precision closely matches the edge-to-edge spatial extent of crowding within $50 \mathrm{~ms}$ before a saccade, and both are approximately half the spatial extent of crowding when no saccade is planned.

sents the endpoint of a saccade included in the critical distance estimates for Experiment 2. We fitted an ellipse that encompassed 95\% of all saccade endpoints (see Materials and Methods). The horizontal extent of the ellipse corresponds to the radial precision of the saccades and the vertical extent denotes the tangential precision. The axes of the fitted ellipse reveal that saccadic precision is poorer in the radial plane than in the tangential plane, with a radial-to-tangential ratio of 1.6. This asymmetry in saccadic precision corresponds closely to the classical radial-tangential asymmetry of critical distance measured using pairs of flankers arranged either radially or tangentially with respect to the target (Toet and Levi, 1992). Moreover, as shown in Figure 5B, the absolute, edge-to-edge spatial extent of crowding when no saccade was planned (gray line) is approximately twice that observed for trials in the $50 \mathrm{~ms}$ just before a saccade (maroon line). Note that oculomotor precision (pink line) closely approximates the spatial extent of crowding just before a saccade.

We further tested the extent to which saccade endpoints are related to identification of a crowded target by comparing performance for trials in which saccades deviated from the target center by more than one degree with those in which the saccade fell within one degree of the target center. We performed this 
analysis separately for three conditions that represent the full range of perceptual performance. We chose (1) the condition in which performance was poorest (100-149 $\mathrm{ms}$ time bin, $1^{\circ}$ targetflanker separation); (2) the condition in which performance improved the most $\left(0-49 \mathrm{~ms}\right.$ time bin, $2^{\circ}$ target-flanker separation); and (3) the condition in which performance was best $\left(0-49 \mathrm{~ms}\right.$ time bin, $5^{\circ}$ target-flanker separation; see Fig. 3). We chose these three conditions to maximize the likelihood of uncovering any effect of saccade accuracy, while limiting the risk of type 1 error due to multiple post hoc comparisons. There was no difference in target discrimination according to saccade accuracy in any of these conditions ( $p s>0.81$, uncorrected), and the differences in performance were $<1.5 \%$ within each condition. Thus, performance corresponds best to the overall precision of eye movements (Fig. 5A) rather than the accuracy of saccade endpoints.

\section{Discussion}

We have shown that improvements in perception immediately before saccade onset, previously demonstrated with isolated stimuli in sparse displays (Remington, 1980; Kowler et al., 1995; Deubel and Schneider, 1996; Moore and Armstrong, 2003; Deubel, 2008), can also operate to release peripheral targets from visual crowding. By systematically varying target-flanker separations, we also found that the critical distance of crowding established during passive fixation shrinks by approximately half in the $50 \mathrm{~ms}$ immediately before saccade onset. The changes in crowding we observed during saccade trials relative to no-saccade trials cannot be attributed to differences in the retinal locations of target and flanker stimuli, as the critical visual events were offset before any eye movements. Moreover, in both saccade and nosaccade conditions observers always knew the precise location of the upcoming target, indicating that the saccade-related mitigation of crowding cannot be ascribed to differences in advance positional information.

The fact that crowding of a target at a fixed retinal eccentricity is significantly attenuated before a saccade implies that extraretinal signals that arise just before the eyes move (Wurtz, 2008) play a key role in modifying the spatial extent of visual crowding, and extend a recent computational modeling report linking crowding and eye movements (Nandy and Tjan, 2012). Enhancement in perceived contrast at the saccade goal (e.g., Rolfs and Carrasco, 2012) might have contributed to the improved discriminability of the crowded targets in our experiments, but our finding that performance improvements varied across target-flanker separations (Fig. 3) suggests that changes in perceived contrast alone cannot account for the results. The change in critical distance just before a saccade (Fig. $4 A$ ) reflects the fact that target identification improved most for the smallest target-flanker separations, but did not change for the larger separations. If the presaccadic benefit we have shown were attributable solely to contrast enhancement of the target, this effect should have been equivalent across all target-flanker separations. Moreover, attempts to mitigate crowding by cueing attention to the target, which has also been linked to increased contrast sensitivity (Carrasco et al., 2000), have not yielded reliable reductions in visual crowding (Scolari et al., 2007; Yeshurun and Rashal, 2010).

Changes in the spatial extent of crowding before a saccade suggest that oculomotor signals required for accurate localization of a saccade target can influence responses of visual neurons involved in integrating form information from the peripheral visual field. Such an influence is consistent with evidence for a close functional relationship between oculomotor and visual sensory areas in the primate brain (Wurtz and Mohler, 1976; Umeno and Goldberg, 1997; Moore et al., 1998; Tolias et al., 2001; Moore and Armstrong, 2003; Moore and Fallah, 2004; Gregoriou et al., 2012). In particular, Tolias et al. (2001) found that just before a saccade there is a reduction in the size of the receptive fields of neurons throughout V4, an area thought to be important in crowding (Anderson et al., 2012), as well as a shift of these receptive fields toward the saccade goal. Such a reduction in the size of receptive fields of V4 neurons could result in a corresponding reduction in the critical distance of crowding. Indeed, the mean reduction in receptive field size reported by Tolias et al. (2001) was $2.1^{\circ}$, similar to the $1.7^{\circ}$ reduction in critical distance we observed just before a saccade (Fig. $4 A$ ). Critically, the changes in receptive field sizes observed by Tolias et al. (2001) were greatest in the final $50 \mathrm{~ms}$ before saccade onset, consistent with our findings in human observers.

The observation that crowding is reduced before a saccade is consistent with recent accounts that suggest that crowding arises from imprecise position information for peripheral stimuli. Greenwood et al., (2009) found that the perceived position of target elements can be accounted for by a weighted average of noisy representations of target- and flanker-feature positions. Following this finding, our results can be explained by assuming that operations involved in preparing and executing an eye movement to a crowded target effectively change the weights of target- and flanker-position noise. For example, weightings for flanker stimuli might be reduced just before oculomotor target selection, via suppression of neural activity in FEF associated with distractor stimuli (Schall et al., 1995). In line with this, saccadic accuracy in Experiment 2 was unaffected by the separation between target and flanking stimuli (Fig. 5A). Alternatively, an impending saccade to a crowded target could simply reduce the position noise of the target via a reduction in the size of the receptive fields of V4 neurons that represent the target (Tolias et al., 2001). Performance for crowded targets is well modeled using values for position and identity uncertainty of unflanked targets (van den Berg et al., 2012), suggesting that sharpening of target position estimates could form the basis for reduced crowding before saccades.

Previous studies have linked perceptual enhancements during saccade and nonsaccade conditions to common neural activity in such areas as FEF and posterior parietal cortex, both of which are known to play an active role in saccade generation (Corbetta and Shulman, 2002). Indeed, changes in the gain of V4 neurons occur even when FEF activity is below that required to trigger a saccade (Moore and Armstrong, 2003; Moore and Fallah, 2004). In line with these accounts, both the elliptical shape and radialtangential asymmetry of saccadic precision we observed in Experiment 2 (Fig. 5) correspond to the classic spatial characteristics of crowding zones measured previously (Toet and Levi, 1992). These observations suggest a functional interaction between oculomotor control systems and visual neurons whose responses are susceptible to crowding in the absence of eye movements. Crucially, our data also reveal that the substantial gains in target discriminability and reductions in critical distance for saccade targets arise exclusively in the last $50 \mathrm{~ms}$ before a saccade, consistent with recent psychophysical reports for uncrowded stimuli (Deubel, 2008; Rolfs and Carrasco, 2012; Rolfs et al., 2011). The time course of these perceptual changes raises the possibility that, in addition to the benefits associated with target selection alone, visual centers involved in oculomotor preparation and execution can modulate visual sensitivity during the immediate presaccadic period (but see Gregoriou et al., 2012). 
An interesting finding in Experiment 2 was that the spatial extent of crowding observed just before a saccade corresponded closely with the overall precision of saccades (Fig. 5). Yet, observers' ability to identify the target did not vary according to the accuracy of saccade endpoints. This finding suggests that changes in crowding during saccade preparation do not simply depend on the specific outcome of the saccade motor command. In our experiment, presaccadic changes in the identification of a target at $7^{\circ}$ in peripheral vision were relatively uniform when saccades landed within $2^{\circ}$ of the target. These data show that the benefits from saccade preparation accrue to a region around the intended endpoint and are not determined by the precision of the executed saccade.

In summary, the current findings are consistent with the idea that oculomotor signals associated with intended eye movements can alter the resolution of object identification within a spatial zone immediately surrounding a peripheral saccade target. This hypothesized link between oculomotor control and visual perception is further supported by the finding that the distribution of saccade errors closely matches the characteristic, radially biased spatial region within which crowding occurs. By using oculomotor signals to release saccade targets from crowding, the visual system may effectively "presample" an object that will soon be foveated for detailed processing. Such a preview of the saccade target may help to explain the subjective experience of visual continuity across eye movements in natural environments where peripheral vision is densely cluttered.

\section{References}

Anderson EJ, Dakin SC, Schwarzkopf DS, Rees G, Greenwood JA (2012) The neural correlates of crowding-induced changes in appearance. Curr Biol 22:1199-1206. CrossRef Medline

Bouma H (1970) Interaction effects in parafoveal letter recognition. Nature 226:177-178. CrossRef Medline

Brainard DH (1997) The Psychophysics Toolbox. Spat Vis 10:433-436. CrossRef Medline

Carrasco M, Penpeci-Talgar C, Eckstein M (2000) Spatial covert attention increases contrast sensitivity across the CSF: support for signal enhancement. Vision Res 40:1203-1215. CrossRef Medline

Corbetta M, Shulman GL (2002) Control of goal-directed and stimulusdriven attention in the brain. Nat Rev Neurosci 3:201-215. Medline

Corbetta M, Akbudak E, Conturo TE, Snyder AZ, Ollinger JM, Drury HA, Linenweber MR, Petersen SE, Raichle ME, Van Essen DC, Shulman GL (1998) A common network of functional areas for attention and eye movements. Neuron 21:761-773. CrossRef Medline

Cornelissen FW, Peters EM, Palmer J (2002) The Eyelink Toolbox: eye tracking with MATLAB and the Psychophysics Toolbox. Behav Res Methods Instrum Comput 34:613-617. CrossRef Medline

Deubel H (2008) The time course of presaccadic attention shifts. Psychol Res 72:630-640. CrossRef Medline

Deubel H, Schneider WX (1996) Saccade target selection and object recognition: evidence for a common attentional mechanism. Vision Res 36: 1827-1837. CrossRef Medline

Efron B, Tibshirani R (1993) An introduction to the bootstrap. New York: Chapman and Hall.

Felisbert FM, Solomon JA, Morgan MJ (2005) The role of target salience in crowding. Perception 34:823-833. CrossRef Medline

Greenwood JA, Bex PJ, Dakin SC (2009) Positional averaging explains crowding with letter-like stimuli. Proc Natl Acad Sci U S A 106:13130-13135. CrossRef Medline

Gregoriou GG, Gotts SJ, Desimone R (2012) Cell-type-specific synchronization of neural activity in FEF with V4 during attention. Neuron 73:581594. CrossRef Medline
Hunt AR, Cavanagh P (2011) Remapped visual masking. J Vis 11(1):13 1-8. CrossRef Medline

Kowler E, Anderson E, Dosher B, Blaser E (1995) The role of attention in the programming of saccades. Vision Res 35:1897-1916. CrossRef Medline

Levi DM (2008) Crowding-an essential bottleneck for object recognition: a mini-review. Vision Res 48:635-654. CrossRef Medline

Moore T, Armstrong KM (2003) Selective gating of visual signals by microstimulation of frontal cortex. Nature 421:370-373. CrossRef Medline

Moore T, Fallah M (2004) Microstimulation of the frontal eye field and its effects on covert spatial attention. J Neurophysiol 91:152-162. Medline

Moore T, Tolias AS, Schiller PH (1998) Visual representations during saccadic eye movements. Proc Natl Acad Sci U S A 95:8981-8984. CrossRef Medline

Moore T, Armstrong KM, Fallah M (2003) Visuomotor origins of covert spatial attention. Neuron 40:671-683. CrossRef Medline

Nandy AS, Tjan BS (2012) Saccade-confounded image statistics explain visual crowding. Nat Neurosci 15:463-469, S1-S2. CrossRef Medline

Parkes L, Lund J, Angelucci A, Solomon JA, Morgan M (2001) Compulsory averaging of crowded orientation signals in human vision. Nat Neurosci 4:739-744. CrossRef Medline

Pelli DG (1997) The VideoToolbox software for visual psychophysics: transforming numbers into movies. Spat Vis 10:437-442. CrossRef Medline

Pelli DG (2008) Crowding: a cortical constraint on object recognition. Curr Opin Neurobiol 18:445-451. CrossRef Medline

Pelli DG, Tillman KA (2008) The uncrowded window of object recognition. Nat Neurosci 11:1129-1135. Medline

Remington RW (1980) Attention and saccadic eye movements. J Exp Psychol Hum Percept Perform 6:726-744. CrossRef Medline

Rolfs M, Carrasco M (2012) Rapid simultaneous enhancement of visual sensitivity and perceived contrast during saccade preparation. J Neurosci 32:13744-13752a. CrossRef Medline

Rolfs M, Engbert R, Kliegl R (2005) Crossmodal coupling of oculomotor control and spatial attention in vision and audition. Exp Brain Res 166: 427-439. CrossRef Medline

Rolfs M, Jonikaitis D, Deubel H, Cavanagh P (2011) Predictive remapping of attention across eye movements. Nat Neurosci 14:252-256. CrossRef Medline

Schall JD, Hanes DP, Thompson KG, King DJ (1995) Saccade target selection in frontal eye field of macaque. I. Visual and premovement activation. J Neurosci 15:6905-6918. Medline

Scolari M, Kohnen A, Barton B, Awh E (2007) Spatial attention, preview, and popout: which factors influence critical spacing in crowded displays? J Vis 7(2):7 1-23. CrossRef Medline

Strasburger H (2005) Unfocused spatial attention underlies the crowding effect in indirect form vision. J Vis 5(11):1024-1037. CrossRef

Toet A, Levi DM (1992) The two-dimensional shape of spatial interaction zones in the parafovea. Vision Res 32:1349-1357. CrossRef Medline

Tolias AS, Moore T, Smirnakis SM, Tehovnik EJ, Siapas AG, Schiller PH (2001) Eye movements modulate visual receptive fields of V4 neurons. Neuron 29:757-767. CrossRef Medline

Umeno MM, Goldberg ME (1997) Spatial processing in the monkey frontal eye field. I. Predictive visual responses. J Neurophysiol 78:1373-1383. Medline

van den Berg R, Johnson A, Martinez Anton A, Schepers AL, Cornelissen FW (2012) Comparing crowding in human and ideal observers. J Vis. 12(6):13 1-14. CrossRef Medline

Watson AB, Pelli DG (1983) QUEST: a Bayesian adaptive psychometric method. Percept Psychophys 33:113-120. CrossRef Medline

Whitney D, Levi DM (2011) Visual crowding: a fundamental limit on conscious perception and object recognition. Trends Cogn Sci 15:160-168. CrossRef Medline

Wurtz RH (2008) Neuronal mechanisms of visual stability. Vision Res 48 : 2070-2089. CrossRef Medline

Wurtz RH, Mohler CW (1976) Enhancement of visual responses in monkey striate cortex and frontal eye fields. J Neurophysiol 39:766-772. Medline

Yeshurun Y, Rashal E (2010) Precueing attention to the target location diminishes crowding and reduces the critical distance. JVis 10(10):16 1-12. CrossRef Medline 